\title{
Response to Francis Murphey's theory on lumbar disc disease: upon reflection
}

\author{
Saeid Alemo
}

Received: 4 January 2011 / Accepted: 5 January 2011 / Published online: 27 January 2011

(C) Springer-Verlag 2011

Dear Editor,

The reviewer is asking whether it is, in fact, independent of Murphey's theory, wise to continue to follow the practice that emerged from Murphey's theory, and if so, why [2]. In my view, medical ethics and cost containment are of concern to the practice of medicine in industrial countries. Intradisc procedures for the treatment of low back pain, including discography and discectomy, are, based on our assessment, experimental and should not be performed [1].

Research should be based on thorough knowledge of the scientific background and a careful assessment of risks and benefits, should have a reasonable likelihood of benefit for the population studied, and should be conducted by suitably trained investigators using approved protocols, subject to independent ethical review and oversight by a properly convened committee [4].

Furthermore, the annual cost of lower back pain in industrial countries is astronomical [3]. The elimination of unnecessary procedures, such as discography and the intradiscal procedures that ultimately have been a burden on taxpayers, is the objective of governments and healthcare providers in industrial countries.

Conflict of interest None.

\section{References}

1. Alemo S, Sayadipour A (2010) Role of facet arthropathy and discopathy in chronic mechanical lower back pain. Neurolog Orthop Med Surg 28:1-7

2. Alemo S, Sayadipour A (2010) Sources and patterns of pain in lumbar disc disease: revisiting Francis Murphey's theory. Acta Neurochir 152:1555-1558

3. Dagenais S, Caro J, Haldeman S (2008) A systematic review of low back pain cost of illness studies in the United States and internationally. Spine J 8:8-20

4. World Medical Association. World Medical Association declaration of Helsinki: ethical principles for medical research involving human subjects. Amended by the 59th WMA General Assembly, Seoul, October 2008. At http://www.wma.net/en/30publications/ 10policies/b3/17c.pdf. Accessed 3 Jan 2011
S. Alemo $(\bowtie)$

Department of Neurosurgery,

Drexel University College of Medicine,

2630 Holme Avenue,

Philadelphia, PA 19152, USA

e-mail: drsalemo@aol.com 